\title{
Editorial
}

\section{Busca de informações sobre o novo coronavírus no Brasil: uma análise da tendência considerando as buscas online}

A

pandemia provocada pelo novo Coronavírus se espalhou rapidamente de forma transcontinental em poucas semanas, e foi declarado em 30 de janeiro de 2020 como uma emergência de saúde pública de interesse internacional pela Organização Mundial da Saúde (OMS). ${ }^{(1)}$ Cientificamente nomeado como SARS-CoV-2 e causador da doença intitulada COVID-19, o vírus foi relatado pela primeira vez em Wuhan, província de Hubei, República Popular da China, no final de dezembro de 2019. ${ }^{(2)}$

A COVID-19 é uma doença respiratória, transmitida por inalação de gotículas lançadas através da tosse ou espirro, e o contato, que se dá por meio do contato com as mucosas bucais, nasais e oculares contaminadas. ${ }^{(3)}$ Por se tratar de uma doença com propagaçáo de natureza exponencial, a OMS e o Ministério da Saúde (MS) propuseram a adoção de medidas a fim de mitigar os efeitos sociais e de saúde da COVID-19, por meio do isolamento social, utilização dos Equipamentos de Proteção Individual, estratégias não farmacêuticas, como a lavagem das mãos, até que se concretizem uma forma efetiva de tratamento, com escopo de prevenir a populaçáo contra a doença e a morte. ${ }^{(4)}$

Com comportamento errático e de rápida disseminação pelo mundo, a COVID-19 contabilizou 9.826 casos confirmados durante o mês de janeiro, 85.403 casos em fevereiro, 750.890 casos em março, chegando a 3.090.445 durante o mês de abril e 6.120 .740 em maio, sendo responsável até o momento por 369.593 mortes (captura dos dados: $16 \mathrm{~h} 55 \mathrm{~m} 15 \mathrm{~s}$ do dia 31 de maio de 2020). ${ }^{(5)}$

No Brasil, o primeiro caso de COVID-19 foi confirmado em 26 de fevereiro de 2020. Os dois primeiros casos confirmados foram de indivíduos do sexo masculino, residentes na cidade de São Paulo, que haviam regressado de viagem à Itália. ${ }^{(6)}$ Após três meses do primeiro caso, o país passou a ocupar a segunda posição entre os países mais infectados do mundo em número de casos confirmados, atrás apenas dos Estados Unidos, e a quarta posição em número de óbitos pela doença, atrás dos Estados Unidos, Reino Unido e Itália. ${ }^{(5)}$ As análises de casos confirmados são particularmente úteis para inferir parâmetros epidemiológicos importantes, como os períodos de incubação e infecciosos e surtos em andamento ou uma probabilidade de surto. ${ }^{(6)}$

Neste contexto, o Google Trends surge como uma fonte de dados que podem ser usados para buscar uma melhor compreensão sobre o interesse público durante este surto pandêmico. ${ }^{(7)}$ Dado o sério impacto do COVID-19 e na direçáo do uso de novos métodos e abordagens para a divulgação e previsão desta pande- 
mia, os dados do Google Trends podem ser usados para explorar a relação entre $\mathrm{o}$ interesse online e eventos marcantes como início da quarentena e aumento no número de óbitos. Atualmente, medidas de infodemiologia (epidemiologia da informação), especialmente se combinadas com dados tradicionais, podem ser parte integrante da vigilância do vírus em nível regional. ${ }^{(8)}$

Diante disso, realizamos um estudo com objetivo de analisar o comportamento de buscas online de informaçôes sobre o novo coronavírus no Brasil e sua relação com as incidências de casos e número de óbitos por COVID-19.

Os dados referentes às buscas na internet relacionadas ao novo coronavírus foram extraídos pelo Google Trends (GT) (https://trends.google.com.br/ trends/). O GT é uma ferramenta de acesso público que apresenta frequências de buscas no Google em um período, permitindo assim dimensionar tendências de buscas sobre temas de interesse discriminadas em delimitaçóes geográficas (estados; países; ou a nível mundial); categorias temáticas (saúde; ciência; notícias; viagens entre outras) além de recortes temporais (períodos específicos; últimos cinco anos; última semana, etc). ${ }^{(7)} \mathrm{O}$ GT não apresenta os valores em números absolutos de buscas, mas os algoritmos do GT normalizam os resultados dos volumes de busca a partir da maior quantidade de buscas para um determinado momento, em uma escala que oscila entre 0 (volume de buscas menor que $1 \%$ em relação ao pico de popularidade) e 100 (pico de acessos) apresentados como Volumes Relativos de Buscas (VRB). ${ }^{(7)}$

O Google Trends vem sendo usado para análises de tendências sobre temas ligados à pandemia de Covid-19 em diversos países como Irã ${ }^{(9)}$ EUA, ${ }^{(10,11)}$ Taiwan, ${ }^{(12)}$ França, ${ }^{(13)}$ China, ${ }^{(14)}$ Itália $^{(15)}$ bem como abordagens comparativas sobre comportamentos de busca em diferentes continentes. ${ }^{(16-18)}$ A metodologia empregada pelo GT é detalhadamente descrita no seu website, ${ }^{(7)}$ assim como em diversos outros estudos. ${ }^{(11,13-15,17-21)}$

Nesta pesquisa, o perfil de buscas explorado foi configurado considerando 72 dias desde o primeiro caso confirmado. Portanto, os dados analisados foram referentes ao período de 26 de fevereiro a 08 de maio do corrente ano, com filtros de região "Brasil" e tópico do assunto "saúde". Os dados foram importados como arquivos CSV (Comma-separated values) para planilhas eletrônicas $\left(\mathrm{MS}\right.$ Excel $\left.^{\circledR}\right)$.

Os termos de buscas analisados foram selecionados com base em revisóes bibliográficas ${ }^{(22)}$ e a partir de observações empíricas e documentais sobre expressôes predominantemente usadas pelo MS e na mídia televisiva nacional para se referir à pandemia. Os termos inicialmente testados e comparados no GT foram "coronavírus", "novo coronavírus" e "Covid-19", sendo que os dois últimos tiveram pouca expressividade na abordagem comparativa, assim, adotou-se o termo "coronavírus". Contudo, vale ressaltar que o GT permite uma análise mais acurada do interesse de busca por meio de tópicos de interesse, pois consolidam diversos termos de busca relacionados de acordo com interpretação dos algoritmos do Google. A grafia do termo influencia na definição do termo, embora no tópico já considere termos relacionados a um mesmo tópico. Sendo assim, também foi realizado um estudo considerando o tópico "coronavírus", que engloba o termo de busca "coronavírus" e outros relacionados. 
Com os resultados apresentados pelo GT, verificou-se que a utilizaçấo do termo sem acento apresentou um maior volume de buscas em relação à grafia com acento agudo, embora notou-se que com o passar do tempo, o termo de busca com acento ganhou mais volume. A figura 1 ilustra uma comparaçáo entre os volumes de busca dos termos "coronavírus" com e sem acento agudo.

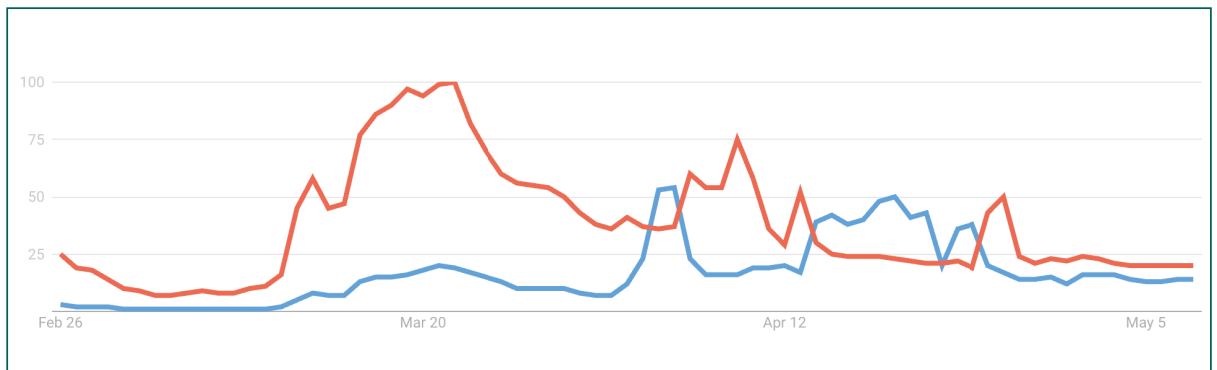

Figura 1. Volume de buscas normalizado dos termos de busca "coronavirus" (vermelho) e 0 termo considerando uma grafia com acento gráfico "coronavírus" (azul)

Em relação ao tópico "coronavírus", verificou-se que o termo de busca "coronavírus" (sem acento) é o principal termo de busca deste tópico. A figura 2 ilustra os volumes de busca do tópico e do termo "coronavírus".

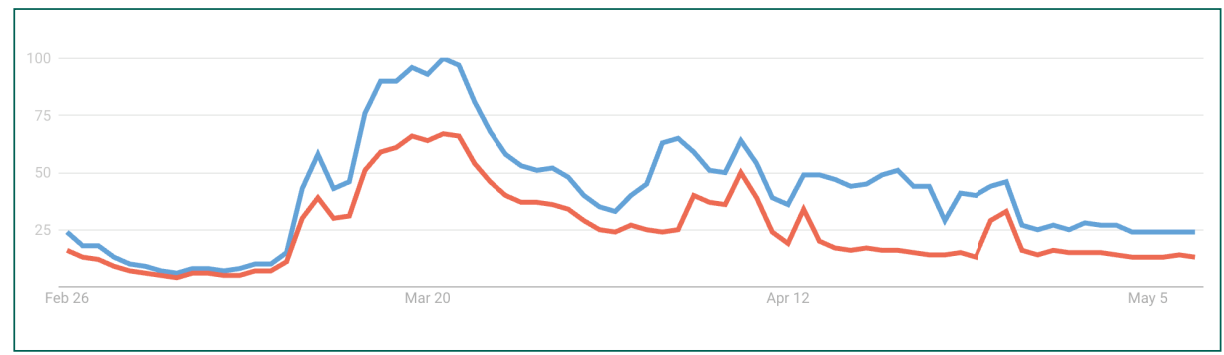

Figura 2. Volume de buscas normalizado do termo de busca "coronavírus" (vermelho) e para as buscas classificadas pelo GT pertencentes ao tópico "coronavírus" (azul)

Ressalta-se que devido a normalização dos dados aplicada pelo algoritmo do GT, o volume máximo de busca nos gráficos reportados será sempre 100 . Comparando os volumes reportados pelo GT, verifica-se que o pico (100) ilustrado na figura 1 , corresponde a $67 \%$ do volume de todos os termos de busca relacionados ao tópico "coronavírus" classificado assim pelos algoritmos do Google como relacionados ao Vírus, conforme ilustrado na figura 2. Ou seja, no pico de interesse, apenas cerca de $33 \%$ das buscas associadas pelos algoritmos Google ao vírus "coronavírus" são outros, além do termo de busca "coronavírus" sem acento.

Outra evidência sobre o comportamento dos usuários na utilização do buscador Google que o GT oferece são os termos de buscas relacionados. A figura 3 apresenta os termos de busca relacionados ao tópico "coronavírus". Novamente, o resultado apresentado com volumes de busca normalizados. As buscas realizadas usando o termo com acento representam apenas $44 \%$ das buscas em relação ao termo "coronavírus" sem acento. Verificou-se tam- 
bém que $21 \%$ das buscas utilizam a forma composta por duas palavras: "corona virus". Portanto, o termo de busca de maior interesse foi a versão sem acento "coronavírus".

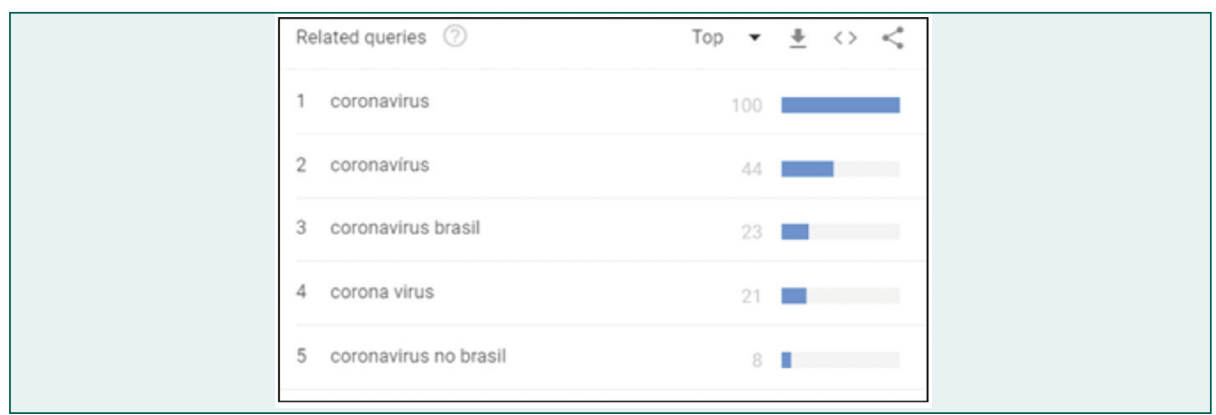

Figura 3. Os cinco termos de busca mais relacionados ao tópico "coronavírus"

Em relação às buscas relacionadas, os algoritmos do GT também identificam padróes de comportamento que são classificados como interesse emergente. Considerando uma classificação pelo interesse emergente, verificou-se que as buscas por dicas de prevenção são de maior interesse, seguido pelo auxílio (governamental) (Figura 4).

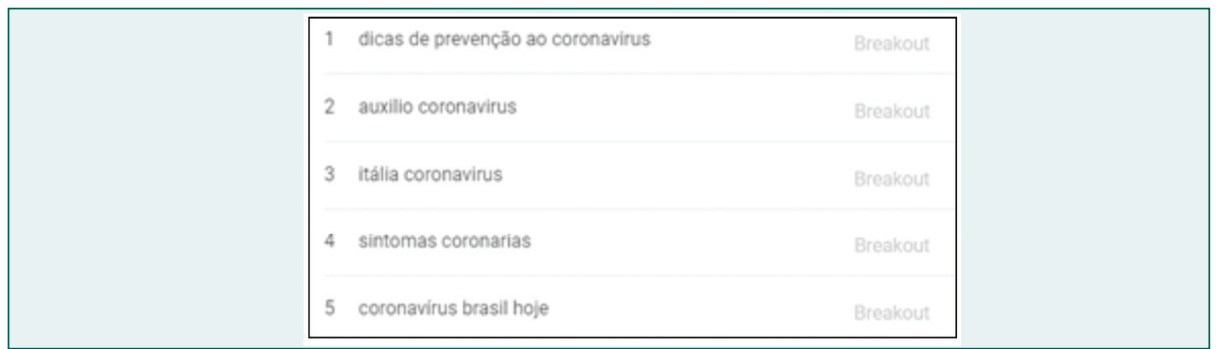

Figura 4. Os cinco termos de busca mais significativos relacionados ao tópico "coronavírus" que são identificados como de interesse crescente pelo GT

No GT é possível buscar uma explicação para o fenômeno, explorando as buscas relacionadas a um termo num período específico. Analisando o gráfico comparativo de interesse entre os termos "coronavírus" com e sem acento, verificou-se que no mês de abril, o termo com "acento" é o mais utilizado. Considerando a análise sobre os termos relacionados, fornecida pelo algoritmo do GT para este período (Figura 5), verificou-se que os usuários buscam informações conjunturais e técnicas sobre o vírus.

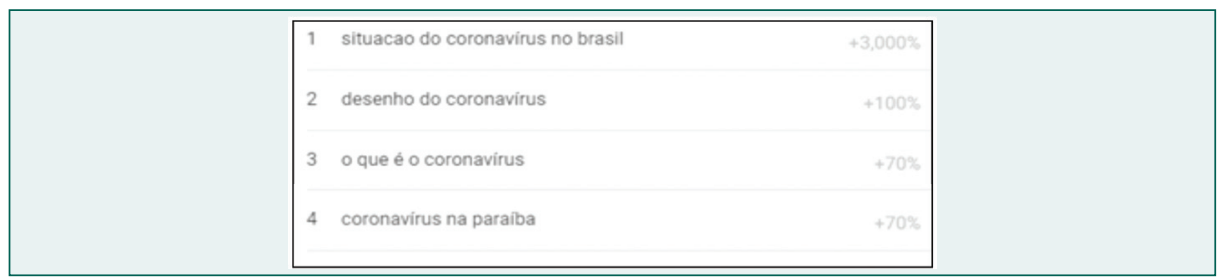

Figura 5. Termos de busca relacionados ao tópico "coronavírus" que são identificados como de interesse crescente pelo GT para o período de 13/04/2020 a 22/04/2020 no Brasil 
Além das evidências apresentadas pelo GT, é importante considerar também na análise a ocorrência e eventos sociais significativos que impactam o comportamento de usuários. O pico de interesse na busca pelo novo vírus coincide ao início da quarentena nas principais capitais do país.

Desta forma, é plausível considerar que a imposição das quarentenas e instauração da situação de emergência pública influenciou significativamente no interesse de busca em diversas regiốes do país. Sendo assim, os usuários buscaram por informaçóes sobre o vírus de forma mais genérica. Já o termo de busca com acento ganhou força a partir de abril, o que pode sinalizar uma maturidade na busca por informaçôes sobre o tema, sendo que um maior número de usuários passou a realizar buscas utilizando o termo com a grafia mais correta - com o acento.

Outro fenômeno interessante observado no interesse dos usuários sobre a questão é a diminuiçấo do volume de busca ao longo do tempo (Figuras 1 e 2). Considerando o fato de o termo de busca com acento ter ganhado força em relação ao termo genérico, pode ser apenas que os usuários passaram a tratar a questáo mais corretamente, realizando buscas com o termo de grafia mais correta. Entretanto, levando em consideração os termos de buscas relacionados, é mais plausível inferir que os usuários se educaram sobre o tema por meio do conteúdo acessado pelas buscas realizadas anteriormente e passaram a buscar por atualizaçôes sobre a situação. $O$ interesse que pode ser entendido como inicialmente em busca de informaçóes a respeito do vírus e a doença, passou para uma fase de acompanhamento, com menos de 1/4 do volume de buscas registrado no pico.

O interesse crescente ao longo do tempo relacionado ao termo de busca com acento "coronavírus", demonstraram o interesse mais educado por atualização conjuntural sobre o desenvolvimento da situação, reforçando a hipótese de que o interesse dos usuários pelo vírus evoluiu de busca por informaçóes, no intuito de se educar para um estágio em que os usuários buscam por atualização em relação à sua ocorrência.

Ademais, neste estudo buscou-se também verificar o impacto no interesse por buscas em relação à notificação dos casos e óbitos decorridos da doença. A partir de dados oficiais disponibilizados pelo MS, foi realizado um estudo estatístico considerando análise descritiva e inferencial dos dados.

As incidências diárias foram extraídas de site oficial do MS (https://covid.saude.gov.br), que é atualizado a partir de dados das secretarias estaduais de saúde, refletindo o avanço das comunicaçôes e óbitos no país relacionados a COVID-19, ainda que alguns estudos salientem a possibilidade de subnotificação dos dados. ${ }^{(22)}$

Para análise estatística foi utilizado o software IBM SPSS ${ }^{\circledast}$, versão 24.0, programa aplicado para a produção dos gráficos de associação entre a evolução das notificaçôes pelo MS e a busca do termo "coronavírus" na plataforma GT e para análise inferencial da correlação entre o número de casos e número de mortes por Covid-19 com o volume relativo de buscas sobre coronavírus no período, por meio do teste de correlação de Pearson.

$\mathrm{Na}$ figura 6, é apresentado o gráfico com o volume de buscas para o tópico "coronavírus" e a quantidade de casos de COVID-19, bem como os 
óbitos registrados no Brasil. Visualmente, é possível perceber que aparentemente não há uma correlação forte entre o interesse de busca e o número de casos ou mortes. A correlação entre os casos identificados e os óbitos é esperada por definição, pois alguns dos casos mais graves da doença vêm a óbito.

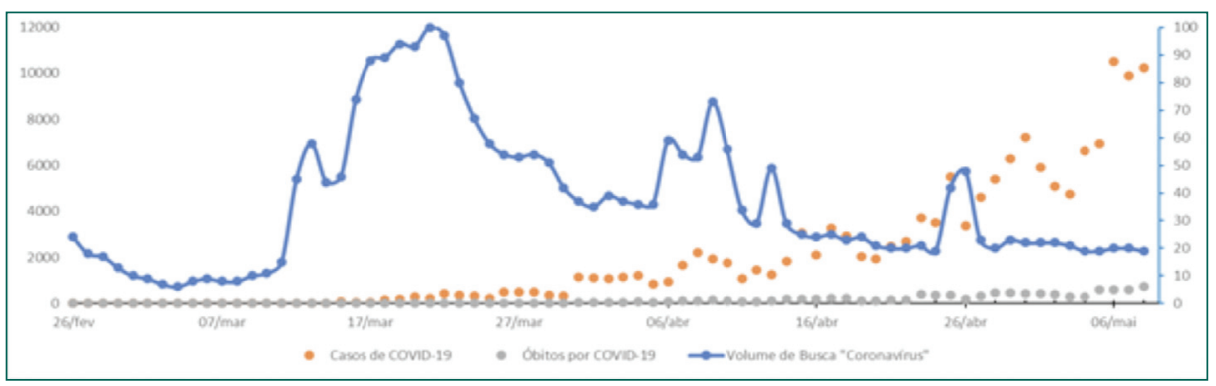

Figura 6. Casos identificados por COVID-19 e óbitos informados pelo MS, e o volume de busca pelo tópico "Coronavirus" reportado pelo GT

Foi realizada uma análise de correlação utilizando a avaliaçáo da relação linear proposta por Pearson. Verificou-se que os casos identificados e os óbitos possuem um coeficiente de correlaçáo de 0,972 , mas a correlação do volume de busca possui um coeficiente negativo, de -0,291, indicando uma relação inversamente proporcional, ou seja, o interesse diminui apesar dos casos aumentarem.

Vale ressaltar que uma correlação não implica em causalidade. A identificação de uma correlação é apenas um indício de que pode haver alguma relação de causalidade entre duas variáveis. A correlaçáo identificada dos casos identificados com os óbitos é por transitividade, visto que a doença leva uma parcela dos casos identificados ao óbito. No caso, de uma relação do aumento de casos com o interesse verificado por meio das buscas, náo foi verificada uma correlaçáa significativa. Esta verificação reforça que o pico de interesse em buscas por informação sobre a situação registrado no volume de buscas no mês de março foi satisfeito, restando um interesse residual por acompanhamento da conjuntura, bem como com picos eventuais motivados, provavelmente, por novos tratamentos, apesar de um crescimento na quantidade de casos.

No que tange aos aspectos éticos, cabe salientar que os dados acessados a partir do GT são de domínio público, o que dispensa a necessidade de submissão da pesquisa a comitês de ética. Desse modo, o estudo foi conduzido de acordo com pressupostos éticos normatizados pela resolução 466/2012 do Conselho Nacional de Saúde.

A utilizaçáo do Google Trends se mostrou efetiva na análise sobre as buscas de termos ligados à pandemia de COVID-19. Em análise comparativa dos termos "coronavírus" com e sem acento, verificou-se que o termo sem "acento" é o mais utilizado, apesar de notável mudança de comportamento no uso destes termos nas buscas. Constatou-se que, diante do início da quarentena, os usuários passaram a realizar buscas utilizando o termo com a grafia mais correta - com o acento, denotando uma mudança para um comportamento mais educado, assim como evidências sobre buscas relacionadas mostram que os usuários se educaram e passaram a buscar informaçôes conjunturais. 
Delmo de Carvalho Alencar (Autor correspondente) ${ }^{1}$ ${ }^{I}$ Escola Nacional de Saúde Pública, Fundação Oswaldo Cruz, Rio de Janeiro, RJ, Brasil https://orcid.org/0000-0002-6555-7921 E-mail:delmo-carvalho@hotmail.com

\author{
Jasilaine Andrade Passos ${ }^{2}$ \\ ${ }^{2}$ Escola Nacional de Saúde Pública, Fundação Oswaldo Cruz, Fiocruz, Rio \\ de Janeiro, RJ, Brasil \\ https://orcid.org/0000-0001-9595-7822
}

Ana Raquel Batista de Carvalho ${ }^{3}$ ${ }^{3}$ Universidade Federal do Piaui, Teresina, PI, Brasil https://orcid.org/0000-0001-5287-1084

\author{
Aline Raquel de Sousa Ibiapina ${ }^{4}$ \\ ${ }^{4}$ Universidade Federal do Piaui, Teresina, PI, Brasil \\ https://orcid.org/0000-0003-1373-3564
}

\begin{abstract}
Dárlinton Barbosa Feres Carvalho ${ }^{5}$ ${ }^{5}$ Pontifícia Universidade Católica do Rio de Janeiro, Rio de Janeiro, RJ, Brasil https://orcid.org/0000-0003-3844-0178
\end{abstract}

\begin{abstract}
Paulo Roberto Vasconcellos-Silva ${ }^{6}$ ${ }^{6}$ Escola Nacional de Saúde Pública, Fundação Oswaldo Cruz, Rio de Janeiro, RJ, Brasil https://orcid.org/0000-0003-4646-3580
\end{abstract}

Como citar: Alencar DC, Passos JA, Carvalho AR, Ibiapina AR, Carvalho DB, Vasconcellos-Silva PR. Busca de informaçôes sobre o novo coronavírus no Brasil: uma análise da tendência considerando as buscas online. Acta Paul Enferm. 2020;33:e-EDT20200004

DOI: http://dx.doi.org/10.37689/acta-ape/2020EDT0004 


\section{Referências}

1. World Health Organization (WHO). Statement on the meeting of the International Health Regulations (2005) Emergency Committee regarding the outbreak of novel coronavirus (2019-nCoV). Geneva: WHO; 2020.

2. Wang C, Horby PW, Hayden FG, Gao GF. A novel coronavirus outbreak of global health concern. Lancet. 2020;395(10223):470-3.

3. Lu CW, Liu XF, Jia ZF. 2019-nCoV transmission through the ocular surface must not be ignored. Lancet. 2020;395(10224):e39.

4. Brasil. Ministério da Saúde. Agência Nacional de Vigilância Sanitária. Orientações Gerais - Máscaras faciais de uso não profissional. Brasília (DF): Ministério da Saúde; 2020.

5. Johns Hopkins Whiting School of Engineering. Center for Systems Science and Engineering. Conornavirus COVID19 Global Cases [Internet]. 2020. [cited 2020 Jun 1]. Available from: https://coronavirus.jhu.edu/ map.html

6. Rafael RM, Neto M, Carvalho MM, David HM, Acioli S, Faria MG. Epidemiologia, políticas públicas e pandemia de Covid-19: 0 que esperar no Brasil? Rev Enferm UERJ. 2020; 28:e49570.

7. Google. Google Trends [Internet]. 2020. [cited 2020 Mai 18]. Available from: https://trends.google.com/trends/

8. Mavragani A. Tracking COVID-19 in Europe: infodemiology Approach. JMIR Public Health Surveill. 2020;6(2):e18941.

9. Ayyoubzadeh SM, Ayyoubzadeh SM, Zahedi H, Ahmadi M, R Niakan Kalhori S. Predicting COVID-19 Incidence through analysis of Google Trends Data in Iran: data mining and deep learning pilot study. JMIR Public Health Surveill. 2020;6(2):e18828.

10. Hong YR, Lawrence J, Williams D Jr, Mainous A 3rd. Population-level interest and telehealth capacity of US hospitals in response to COVID-19: cross-sectional analysis of google search and national hospital survey data. JMIR Public Health Surveill. 2020 Apr;6(2):e18961.

11. Yuan X, Xu J, Hussain S, Wang H, Gao N, Zhang L. Trends and prediction in daily new cases and deaths of COVID-19 in the United States: an internet search-interest based model. Explor Res Hypothesis Med. 2020;5(2):1-6.

12. Husnayain A, Fuad A, Su EC. Applications of Google Search Trends for risk communication in infectious disease management: A case study of the COVID-19 outbreak in Taiwan. Int J Infect Dis. 2020;95:221-3.

13. Kluger N, Scrivener Y. The use of Google trends for acral symptoms during COVID-19 outbreak in France. J Eur Acad Dermatol Venereol. 2020 May 2. doi: 10.1111/jdv.16572.

14. Li C, Chen LJ, Chen X, Zhang M, Pang CP, Chen H. Retrospective analysis of the possibility of predicting the COVID-19 outbreak from Internet searches and social media data, China, 2020. Euro Surveill. 2020;25(10):2000199.

15. Rovetta A, Bhagavathula AS. COVID-19-related web search behaviors and infodemic attitudes in Italy: infodemiological study. JMIR Public Health Surveill. 2020;6(2):e19374.

16. Effenberger M, Kronbichler A, Shin Jl, Mayer G, Tilg H, Perco P. Association of the COVID-19 pandemic with Internet Search Volumes: A Google Trends ${ }^{\text {TM }}$ Analysis. Int J Infect Dis. 2020 ;95:192-7.

17. Strzelecki A. The second worldwide wave of interest in coronavirus since the COVID-19 outbreaks in South Korea, Italy and Iran: A Google Trends study. Brain Behav Immun. 2020;S0889-1591(20)30551-1.

18. Walker A, Hopkins C, Surda P. The use of google trends to investigate the loss of smell related searches during COVID-19 outbreak. Int Forum Allergy Rhinol. 2020 Apr. https://doi.org/10.1002/alr.22580.

19. Vasconcellos-Silva PR, Carvalho DB, Trajano V, de La Rocque LR, Sawada AC, Juvanhol LL. Using Google Trends Data to study public interest in breast cancer screening in brazil: why not a pink february? JMIR Public Health Surveill. 2017;3(2):e17.

20. Shin SY, Seo DW, An J, Kwak H, Kim SH, Gwack J, et al. High correlation of Middle East respiratory syndrome spread with Google search and Twitter trends in Korea. Sci Rep. 2016;6(1):32920.

21. Poletto C, Boëlle PY, Colizza V. Risk of MERS importation and onward transmission: a systematic review and analysis of cases reported to WHO. BMC Infect Dis. 2016;16(1):448.

22. Platero K, Gomes F. Números estatísticos e realidades: Uma proposta de reflexão sobre a pandemia de Covid-19 no Brasil. Dilemas; 2020. p. 1-11. 